\title{
List of Food Substitutes vs Nutrients Balance: Which one is the future of designing diets in $21^{\text {st }}$ century?
}

\author{
Mohammad Reza Shadmand Foumani Moghadam ${ }^{1}$ \\ ${ }^{1}$ Department of Nutrition Sciences (Med-Sci), Varastegan Institute for Medical Sciences, \\ Mashhad, Iran
}

July 7,2021

\begin{abstract}
One of the first lines in controlling diseases and malnutrition is diet therapy and currently, due to tool limitations, for designing the patients' diet plan, the food groups and the list of food substitutes are being used while suitable databases of foods composition are available. Methods of designing individuals' diet and total of 8791 foods are reviewed to calculate the nutrients of mixed foods and design a suitable method of diet therapy with more freedom for the patients in the future. Food groups because of their simplicity are suitable for public health educations but have limitations that could be better covered using nutrient balances. The main cause of developing the list of food substitutes was the limitations in calculating nutrients in a designed diet that currently could be run using applications. Patients also by using self-management nutrition applications that based on the nutrient balancing method shown they are ready to accept this method. A well-designed application can make using nutrient balancing method which is the future of diets possible. However, the main limitation is still the needs for a big, validated, and accurate foods composition database that needs to be developed.
\end{abstract}

Keywords : Diet, Food Groups, Nutrients, Diet therapy, Nutrition

\section{Introduction :}

The importance of following a healthy diet to have a healthier life, controlling diseases and improving the clinical stages of patients is well known (1). However, clinical status can put the patients at a higher risk, losing weight and malnutrition, that nutritional interventions will be necessary $(1,2)$. At the first line of nutritional interventions, consultation and healthy-diet recommendations are suggested while by the severity of the clinical conditions, more interventions like planning diets (diet therapy) and using enteral meals are essential (1). Currently, generally, registered dietitian nutritionist (RDN) for designing the patients' diet plan, use the food groups and the list of food substitutes $(1,3)$ while suitable databases of foods composition are available that could replace the food groups because of their more accuracy. By considering the advantages in past years and the importance of designing better diets, this review aims to introduce a possible suitable method to design individuals' diet in the future.

Method :

After reviewing a total of 8791 foods, a statical method to calculate the nutrients of mixed foods as an inexpensive method was established. A total of 859 traditional mixed foods was recalculated using the new method with more accuracy than the last method. Additionally, methods of designing individuals' diet are reviewed to design a suitable method of diet therapy with more accuracy and freedom for the patients in the future. The possible limitations noticed and considered important to report.

Result and Discussion : 
Using food groups was a long-used method that RDNs are already used to it. My plate and food pyramids are also based on this method that shows its effect on public health nutritional policies (1). The main setting in characterizing foods in groups is their percentage of lipids, protein and carbohydrate while the serving sizes of foods in each group are established based on the energy of each food. Currently, most diet therapies are based on this tool and the works on this method made it known better. The food groups main strength is its ease to understand, however, when it comes to communities with mixed foods or patients with special conditions that nutrients would be critical, this method faced some complications that are not possible to cover. On the other hand, using nutrients and their balance as the reference for designing diets will remove these limitations. However, despite more accuracy of nutrients balance method than food groups especially in nutrients except for energy, lipids, protein, and carbohydrate (table-1), the main limitation in using nutrients balance is the time consuming and detailed process of calculating the planned diet nutrients in compare with total energy expenditure (TEE) and the individuals' required nutrients per day. These days, by the current advantages in computer science, the softwares can run these time consuming and detailed process in a real-time process. The unexpected effect of the food processor and different cooking methods that could affect the density of nutrients (leaking or absorption from/to the environment), digestibility and bioavailability of nutrient (4) also remain as a limitation in both methods but it would have lessor effect in nutrient balance method.

Currently, some popular nutrition softwares are using the balancing method with the aim of nutritional self-management that could be good evidence that society is ready to replace food groups with nutrients balance (at least macronutrients) $(5,6)$. The other outcomes of using this softwares shown not only they are widely accepted tool but also could be effective in the community to control some eating disorders, weight control and depression $(5,7,8)$. However, their assessed effect is limited to public health, eating disorders and weight loss studies. This limitation also made these apps remove some nutrients that may be useful for designing the diet of cardiovascular, kidney, metabolic and allergic patients. The current main limitation about these softwares is that there is neither a good study to assess these apps efficiency in clinical studies nor any available information about their database, especially for mixed foods. However, a study in 2018 suggested the current top 5 applications in this field are tend to report nutrients lower than those from NDSR, a dietary analysis software developed for research purposes, but still possible to be used for the clinical purpose (6). For more developments, still, a reliable, valid food composition database especially with mixed foods is required.

By providing a suitable database and a system to calculate the total of diet nutrients, not only the RDNs will be able to design their patients diet more accurate but also could adjust the minimum and maximum required nutrients/day according to clinical stages of their patients and Dietary Reference Intake (DRI) while leaving the food choosing option to the patients under adjusted limitations. However, it required a highquality double-side relationship through software that could provide both self-management and telemedicine between the patients and their RDN. As reported in table 2, there are benefits and limitations for all these 3 methods of diet therapy but using the nutrient balancing method has good potential to replace food groups that could lead to a higher quality of diets in future of diet therapies. However, there is still a long way to assess its efficiency in improving the quality of diet therapies. Currently, the limitation is the inadequate valid database of mixed foods in different regions that may make this method out of reach at the current stage for some countries.

Conclusion : Food groups because of their simplicity are suitable for public health and healthy diet educations but could not have enough accuracy to design a suitable diet for patients in compare with nutrients balance especially in nations those have mixed food pattern. Currently, applications made everything simpler and a well-designed application that links self-management, designing diet using balancing method and telemedicine is the future of diet plans. However, the main limitation is still the needs for a big, validated, and accurate foods composition database. For this reason, as the first step, developing the mixed and traditional foods databases in all regions is required.

Acknowledgment : I want to acknowledge the departments of nutrition at Varastegan Institute for Medical 
Sciences and Mashhad University of Medical Sciences for their support during the last 3 years.

Funding : This short-review did not receive any fund.

Conflict of Interest: the author declares no conflict of interest. However, the author is the deputy chairman and co-founder of Calorito ${ }^{\circledR}$ nutrition software Co. (Mashhad, Iran) that is assessing the best tool to design diets in the future for patients and obtained intellectual and financial property rights of current thesis. Calorito ${ }^{\circledR}$ because of importance of research and advance in health care system specially in nutrition has no conflict about publishing this short-review.

Contribution : We are currently working on the development of a statistical method to calculate the most accurate nutrients of mixed foods that will be published after final improvements. For those who interested but not able to calculate the nutrients of the food, we sincerely invite them to send their national mixed foods ingredients and recipe to calculate by referring to the USDA database.

Further information: This thesis is under assessment in cooperate with the Mashhad University of Medical science, Varastegan Institute for Medical Science and a group of registered dietitian and nutritionists (RDNs) as the referee board. The current provided information in tables are the important parts of rational aims of running this programme.

\section{References :}

1. Raymond JL, Morrow K. Krause and Mahan's Food and the Nutrition Care Process E-Book: Elsevier Health Sciences; 2020.

2. Bischoff SC, Austin P, Boeykens K, Chourdakis M, Cuerda C, Jonkers-Schuitema C, et al. ESPEN guideline on home enteral nutrition. Clinical nutrition. 2020;39(1):5-22.

3. Cipullo L. Category: Food Substitutes.

4. Gibson RS, Perlas L, Hotz C. Improving the bioavailability of nutrients in plant foods at the household level. Proceedings of the Nutrition Society. 2006;65(2):160-8.

5. Osborne H, Brown R, Taylor R, McArthur J, Meredith-Jones K, Roy M, et al. What monitoring strategies are most successful for promoting weight loss? A randomised controlled trial. The FASEB Journal. 2017.

6. Griffiths C, Harnack L, Pereira MA. Assessment of the accuracy of nutrient calculations of five popular nutrition tracking applications. Public health nutrition. 2018;21(8):1495-502.

7. Linardon J, Messer M. My fitness pal usage in men: Associations with eating disorder symptoms and psychosocial impairment. Eating behaviors. 2019;33:13-7.

8. Hobson G. Use of the My Fitness Pal Mobile Application to Decrease Body Mass Indexes and Improve Depressive Symptoms: Brandman University; 2021.

Table 1. comparison the estimated nutrients of two methods of designing diet plans for a normal 1500 kcal diet using food groups by referring to USDA database (in both same foods are used).

\begin{tabular}{llll}
\hline & Using food groups & Using nutrient balance & food groups/ nutrient balanc \\
\hline Energy (Kcal) & 1565 & 1580.041 & 99 \\
Protein (gr) & 84.5 & 78.1494 & 108 \\
Carbohydrate (gr) & 218.5 & 232.197 & 94 \\
Lipid (gr) & 35 & 39.79703 & 87 \\
Fibre (gr) & 31 & 19.29789 & 160 \\
Fatty acids, total saturated (g) & - & 11.33484 & - \\
Cholesterol (mg) & - & 219.8 & -
\end{tabular}




\begin{tabular}{llll}
\hline & Using food groups & Using nutrient balance & food groups/ nutrient balanc \\
\hline Fatty acids, total trans (g) & - & 0.196462 & - \\
Vitamin A, (IU) & - & 19876.85 & - \\
Iron, Fe (mg) & - & 10.97359 & - \\
Potassium, K (mg) & - & 3084.311 & - \\
Calcium, Ca (mg) & - & 1128.089 & - \\
Sodium, Na (mg) & - & 1054.395 & - \\
& & \\
\hline
\end{tabular}

The current diet is one of thousand diets that had comparable different between two methods. This diet is a close diet to the simple food containing diet pattern and only has one fixed food (Kheer). In most cases, the majority of diets are mixed foods. The current normal 1500 Kcal diet that was designed by a RDNs for weight loss includes 3 loaves of Baguette bread (90gr), 4 spatulas baked rice (262 gr), 1 glass of low-fat cow-milk (244 cc), 1 glass low-fat yoghurt, 1 glass of Kheer (180 gr), 2 boxes of matches size fried chicken (60 gr), 2 boxes of matches size grilled chicken, a banana (118 gr), a cucumber (251 gr), an apple (100), a cluster of grape, 2 carrots (110 gr), and a glass of Sabzi Khordan (Raw mint, parsley, young leek leaves, and radish) (75 gr) equal to 7.5 units grains, 3 units low-fat dairies, 4 units low-fat meats, 3 units fruits, and 5 units vegetables.

Table 2. comparison of advantages between designing diets using food groups and the two methods that could use the nutrient balancing method.

\begin{tabular}{ll}
\hline Criteria & Food groups diet design \\
\hline Need any specific tool & No (just the list of food substitutes) \\
The accuracy to provide the requirements & Acceptable \\
Can consider macronutrients & Yes \\
Can consider micronutrients & No (or few) \\
Database requirement & Not necessary \\
Need education & Need to educate the list of food substitu \\
Duration of education & $10-20$ minutes \\
Can consider mixed foods & No \\
Freedom to choose the foods & According to the list of food substitutes \\
Can be used for special diets that require limitation in some nutrients & No (the list of food substitutes will be \\
Time to review patients record and design a 1-week diet & $45-120$ minutes (without using prepared \\
Best usage & public health and healthy diet education \\
\hline
\end{tabular}

